\title{
Recommendations for calibration factors for a photo-reference method for aerosol Black Carbon concentrations
}

Vipul Lalchandani ${ }^{a}$, Sachida N. Tripathi ${ }^{*},{ }^{,}$, Eric Graham ${ }^{b}$, Nithya Ramanathan ${ }^{c}$, James J. Schauer ${ }^{d}$ and Tarun Gupta ${ }^{a}$

*, a Department of Civil Engineering, Indian Institute of Technology, Kanpur 208016, India

${ }^{\mathrm{c}}$ Center for Embedded Networked Sensing, University of California, Los Angeles, 3563 Boelter Hall, Los Angeles, CA 90095-1596.

${ }^{\mathrm{b}}$ Nexleaf Analytics, 2356 Pelham Ave., Los Angeles, CA 90064, USA

${ }^{\mathrm{d}}$ Environmental Chemistry and Technology Program, Wisconsin State Laboratory of Hygiene, University of Wisconsin-Madison, Madison, WI 53706, USA

Correspondence: snt@iitk.ac.in

Contact: +91-5122597845

Fax: $+91-512-2597395$

\begin{abstract}
Large measurement networks of Black Carbon (BC) aerosol are important for understanding its impacts on climate and health. PM2.5 filter samples were collected at three urban US locations and one India urban location and were analyzed for Elemental Carbon (EC) and Organic Carbon (OC) concentrations using thermo-optical analysis (TO) following the IMPROVE protocol for US samples and NIOSH protocol for India samples. Site and season-specific calibrations of an inexpensive photo-reference (PR) method were created with TO EC measurements of the US filter samples whereas method-specific calibration was prepared using India filter samples. Piece-wise calibration based on filter loading was also explored. Calibrations were applied across different sites, seasons and methods to determine Root Mean Square Error (RMSE) and average absolute error in each calibration by comparing with reference EC measurements. This paper investigates various calibrations of PR method to improve the agreement between PR method and TO EC measurements. Difference in BC estimated error remained within $\pm 10 \%$ among three urban US site-specific calibrations, which suggests that site-specific calibrations are
\end{abstract}


not necessary. Season based calibrations were found to perform best (least RMSE/Mean EC), when applied to same season test samples but resulted in large errors of up to $60 \%$ RMSE/Mean EC when applied to different seasons, thus warranting the use of season-specific calibrations of the PR method. RMSE relative to mean EC was 50\% when calibration prepared from US samples (IMPROVE protocol) was used to test India samples (NIOSH protocol). However, method-specific calibration prepared from India samples reduced the error to $24 \%$, showing the large dependency of PR method on reference BC measurement method. Calibration based on filter loading reduced the RMSE slightly for both US urban and India samples and indicated that filters with loadings higher than $20 \mu \mathrm{g} \mathrm{cm}^{-2}$ are not suitable for estimating BC by PR method.

Keywords: Photo-reference method; Elemental Carbon; site-specific calibration; season-specific calibration; method-specific calibration.

\section{Introduction}

Large scale measurement of Black Carbon (BC), often referred to as Elemental Carbon (EC), is important for properly understanding its effects on global and regional climate change, and health. $\mathrm{BC}$ is formed primarily by incomplete combustion and directly absorbs sunlight, heating the particles and the immediate atmosphere (Schwartz and Buseck, 2000).It has been estimated that the direct effect of $\mathrm{BC}$ is the second most important contributor to global warming (Jacobson, 2001)and the climate forcing importance of BC has been underscored by numerous studies (Ramanathan and Carmichael, 2008; Grieshop et al., 2009; Ramanathan and Feng, 2009).BC also impacts health and visibility (Samet et al., 2000; Pope et al., 2002) with the inhalation of smoke containing $\mathrm{BC}$ responsible for an estimated 1.8 million deaths per year (Ezzati and Kamen, 2002). 
The measurement of $\mathrm{BC}$ is important for the evaluation of air pollution and climate models and to evaluate the effectiveness of $\mathrm{BC}$ mitigation programs. The use of terms $\mathrm{BC}$ and EC is dependent on the measurement method as the former is defined optically using aerosol light absorption whereas latter is defined thermally using its refractory properties. $\mathrm{BC}$ can be measured using different optical and mass based instruments (Cross et al., 2010), although results are method-specific and can differ widely (Hitzenberger et al., 2006). The Aethalometer (Hansen et al., 1984) and other filter based methods (Bodhaine, 1995) measure BC during or after loading on a filter through the attenuation of light of accumulating particles, whereas thermo-optical (TO) systems measure Elemental Carbon (EC) by combustion through a series of temperature ramps with an optical correction procedure for charring of organic compounds during pyrolysis (Schmid et al., 2001). Different protocols have been developed to determine the Elemental Carbon (EC) - Organic Carbon (OC) split point in TO analyzers. Two such methods, NIOSH and IMPROVE which are used in this study, have the same thermal evolution method but different temperature and optical monitoring methods (Chow et al., 2001). It has been shown that the two methods result in equivalent Total Carbon (TC) values whereas EC values obtained from NIOSH are typically less than half of those obtained from IMPROVE protocol. Primary difference between the two protocols as shown by Chow et al. (2001) is the allocation of carbon evolved at $850^{\circ} \mathrm{C}$ in NIOSH to OC rather than EC, which when corrected results in good agreement between the two methods.

The cost of such measurement systems can be prohibitive for multi-location, large scale experimental or monitoring efforts. Recently, a new photo-reference (PR) method has been developed for measuring black carbon based on photographs of exposed, aerosol loaded filters that have been placed on a calibration chart that contains reflectance standards associated with 
known $\mathrm{BC}$ concentrations. The method is based on the fact that Black Carbon loading on the filter is tightly correlated with red color pixel or red reflectance (R) value of the filter image, which decreases as the BC loading on the filter increases (Ramanathan et al., 2011).Red reflectance of a filter image is expressed in RGB (red-green-blue) color space and ranges from 0 (pure black) to 255 (pure white). The method works with any digital colorimeter or imageforming instrument, including inexpensive mobile phone cameras. The PR method has been calibrated to Aethalometer and TO instruments and is significantly less expensive than other BC measurement methods. The PR method is also relatively easy to follow in field conditions for personnel with a minimum amount of training and provides rapid $\mathrm{BC}$ measurements within about $20 \%$ of the calibrated standards (Ramanathan et al., 2011).As per our communication with the researchers in atmospheric science and air pollution assessment, the use of PR method is increasing where the access and resources for EC-OC analysis are not possible. The potential sources of error in the method are the digital imaging devices having different color correction algorithms and change in the lighting and exposure while creating the images, which can alter the actual darkness of the filter, and thus change the red reflectance value. To account for this, a reference scale having $\mathrm{BC}$ calibration standards is included in each image to calibrate for different ambient conditions, and is described in detail in our earlier publication (Ramanathan et al., 2011).

The objective of the present work is to examine the difference in the correlation of thermo-optical EC loading with the PR method using aerosol samples collected indifferent sites and seasons. It is hypothesized that site-, season- and method-specific calibrations would help in better understanding the correlation of red reflectance and EC loading, and thus could be used to reduce error of the PR method for large scale BC monitoring purposes. Calibrations based on the 
loading of the filters were also hypothesized to improve BC estimates. US samples were used to create site-, and season-specific calibrations and a combined calibration using all US samples following IMRPOVE protocol was used to predict BC for Kanpur samples and were tested with TO-EC measurements following NIOSH protocol. Finally, the calibration constructed from Kanpur samples following NIOSH protocol was used for predicting BC for the same samples to determine if a method specific calibration can reduce the error in prediction. Interactions between calibrations were not considered in order to minimize the complexity of analysis.

\section{Methodology}

\subsection{Filter Sampling}

Two sets of aerosol loaded filters were used in this study: One at IIT Kanpur campus which is located in the industrial city Kanpur, India $\left(26.5^{\circ} \mathrm{N}, 80.3^{\circ} \mathrm{E}\right)$ and the other from three US urban cities: Los Angeles $\left(34.1^{\circ} \mathrm{N}, 118.25^{\circ} \mathrm{W}\right)$ and Riverside $\left(33.9^{\circ} \mathrm{N}, 117.4^{\circ} \mathrm{W}\right)$, California, and Denver $\left(39.7^{\circ} \mathrm{N}, 104.9^{\circ} \mathrm{W}\right)$, Colorado.

In IIT Kanpur, a locally designed impaction based PM2.5 sampler (Gupta et al., 2011) and a high volume sampler were used to collect PM2.5 samples on $47 \mathrm{~mm}$ diameter (Whatman, QMA) and A4 size quartz filters, respectively, during January 16, 2010 to February 20, 2010. Filters were preconditioned at $550^{\circ} \mathrm{C}$ in an oven to evaporate any adsorbed carbon present in the filter prior to sampling. Flow rate of the sampling was 15 litres per minute (lpm) in case of 47 $\mathrm{mm}$ filters and $1000 \mathrm{lpm}$ in case of A4 size filters. A total of six samples were collected in a day: one in morning from 7:30 a.m. to 10:30 a.m., three in the day time from 11:00 a.m. to 11:30 p.m. (one every $2.5 \mathrm{hrs}$ ) with 30 min gap in between for filter change. Samples were sealed in plastic containers and refrigerated at $4^{\circ} \mathrm{C}$ to avoid any loss of particles until the analysis was performed (Kaul et al., 2011). 
Quartz filters from urban US locations were collected by stations maintained by the Environmental Protection Agency to collect urban BC aerosols. A total of 333 PM2.5 filter samples were collected which includes 116 from LA, 96 from Denver and 121 from Riverside. Samples were collected on $25 \mathrm{~mm}$ quartz filter, using a sampler made by URG (URG-3000N). The sampling was done from midnight to midnight, approximately every $3^{\text {rd }}$ day for a year in 2011. Flow rate of the sampling was $22 \mathrm{lpm}$. Filters were provided for analysis by the Desert Research Institute, Nevada System of Higher Education.

\subsection{Measurement of $\mathrm{BC}$}

Filters from urban India (IIT Kanpur) were analyzed for EC and OC loadings using a TO analyzer (Sunset Laboratory) following NIOSH5040 TOT protocol (NIOSH, 1996). Filters from urban US were also analyzed using a TO analyzer (Sunset Laboratory) following the IMPROVE TOT protocol (Chow et al., 2001). EC and OC were reported as surface loading $\left(\mu \mathrm{g} \mathrm{cm}^{-2}\right)$. Instrument detection limit for the analysis is $\sim 0.05 \mu \mathrm{g} \mathrm{cm}$. EC loading $\left(\mu \mathrm{g} \mathrm{cm}^{-2}\right)$ can be converted to EC concentration $\left(\mu \mathrm{g} \mathrm{m}^{-3}\right)$ by the following equation:

$$
\text { EC loading } \mu \mathrm{gcm}-2=\mathrm{EC} \mu \mathrm{gm}-3 . \mathrm{F} . \mathrm{TA}
$$

where $\mathrm{F}$ is the volumetric flow rate $\left(\mathrm{m}^{3} \mathrm{~min}^{-1}\right), \mathrm{T}$ is the time duration $(\mathrm{min})$, and $\mathrm{A}$ is area of the filter $\left(\mathrm{cm}^{2}\right)$.

\subsection{Creating site-, season-, method-, and loading-specific calibrations}

A random set of 39 samples were selected each from $\mathrm{LA}(\mathrm{n}=116$; mean $\mathrm{EC} \pm \mathrm{SD}=8.75 \pm$ $\left.6.24 \mu \mathrm{g} \mathrm{cm}^{-2}\right)$, Denver $\left(\mathrm{n}=96\right.$; mean $\left.\mathrm{EC} \pm \mathrm{SD}=5.50 \pm 3.66 \mu \mathrm{g} \mathrm{cm}^{-2}\right)$ and Riverside $(\mathrm{n}=121 ;$ mean $\mathrm{EC} \pm \mathrm{SD}=6.77 \pm 5.11 \mu \mathrm{g} \mathrm{cm}^{-2}$ ) data sets to construct site-specificcalibrations.EC loading range 
covered was largest in case of LA (1.42-32.55) $\mu \mathrm{g} \mathrm{cm}^{-2}$, whereas comparable for Denver (0.6415.80) $\mu \mathrm{g} \mathrm{cm}^{-2}$ and Riverside (1.10-22.67) $\mu \mathrm{g} \mathrm{cm}^{-2}$. The optical reflectance as quantified by red pixel value of the filter image (PR method; Ramanathan et al., 2011), were determined using a hand-held spot colorimeter (i1 Basic Pro, X-Rite, Inc. USA). An exponential calibration curve of red reflectance versus EC $\left(\mu \mathrm{g} \mathrm{cm}^{-2}\right)$ was constructed for all three cities. A combined calibration was also constructed by combining the data of three cities $(n=333)$ and will be referred further in the text as "US combined" calibration. Each city's calibration was applied to test data set of each city (not included in creating calibrations): LA ( $\mathrm{n}=77$; mean $\mathrm{EC} \pm \mathrm{SD}=8.33 \pm 5.87 \mu \mathrm{g} \mathrm{cm}^{-2}$ ), Denver $\left(\mathrm{n}=57\right.$; mean $\left.\mathrm{EC} \pm \mathrm{SD}=5.83 \pm 3.63 \mu \mathrm{g} \mathrm{cm}^{-2}\right)$ and Riverside $(\mathrm{n}=82$; mean $\mathrm{EC} \pm \mathrm{SD}=$ $\left.6.72 \pm 5.15 \mu \mathrm{g} \mathrm{cm}^{-2}\right)$. BC estimates in $\mu \mathrm{g} \mathrm{cm}^{-2}$ from each calibration were compared to EC loadings measured using EC-OC analyzer. To analyze the difference in $\mathrm{BC}\left(\mu \mathrm{g} \mathrm{cm}^{-2}\right)$ estimates between calibrations, average absolute error and root-mean-square-error (RMSE) were calculated for each comparison. RMSE is used to combine the magnitude of errors for a given data set into a single metric. It is defined as the square root of the mean of the square of individual differences (true value - predicted value) whereas average absolute error is the mean of the absolute value of the individual differences. RMSE is divided by mean of the EC loadings for the particular data set to normalize the varying range of EC in different data sets.

Combined data of the three cities (LA, Denver and Riverside) for the entire year (2011) was divided into four seasons: spring (March-May), summer (June-Aug), fall (Sep-Nov) and winter (Dec-Feb). From each season data set, 39 sample points were selected by selecting 13 samples from each month to create season-specific calibrations as described above.

For IIT Kanpur samples $\left(\mathrm{n}=72\right.$; mean $\mathrm{EC} \pm \mathrm{SD}=2.05 \pm 1.51 \mu \mathrm{g} \mathrm{cm}{ }^{-2} ; \mathrm{EC}$ range $=(0.48-$ 8.47) $\mu \mathrm{g} \mathrm{cm}^{-2}$, a random set of 40 PM2.5 filter samples were selected as a training set of filters. 
An image of a $2.5 \mathrm{~cm}$ diameter cut-out of each filter was digitally created using a flatbed photo scanner (HP Scanjet 4370) instead of camera to reduce the variations in lighting conditions. From the resulting digital images, the optical reflectance, as quantified by the red pixel value (Ramanathan et al., 2011), was averaged using photo editing software within the area of each filter (approximately $3 \mathrm{~cm}^{2}$ ) to reduce inhomogeneities in $\mathrm{BC}$ accumulation and filter irregularities. An exponential calibration curve of optical reflectance was then constructed between red reflectance and EC loading on the filter measured using EC-OC analyzer following NIOSH protocol.

The entire data sets from the US urban samples and the India samples were used separately to determine if piece-wise regression would improve BC estimates. Loading values, based on red pixel reflectance values, were fit with a two-part and three-part, piece-wise regressions (linear, exponential, and quadratic), using quantile fits in order to reduce the effect of outliers in the data. Additionally, the loading value of the "knot" that joined the two or three regressions was chosen as an optimization of quantiles. Normal quantile-quantile (Q-Q) plots were generated for each transect as a first-pass for estimating goodness of fit.

Regressions using least squares method of curve fitting and statistical tests were performed using Origin (Origin Lab Corporation, USA). Piece-wise regression analysis was performed using the freely available statistical analysis program $\mathrm{R}$ (version 2.13.1; $\mathrm{R}$ Development Core Team, 2011).

\section{Results and discussions}

\subsection{Site-specific calibrations}

EC surface loading was found to have a strong exponential relationship between red reflectance $(\mathrm{R})$ of the loaded filter with a correlation coefficient of $0.89,0.91,0.86$ and 0.87 for 
LA, Denver, Riverside and US combined, respectively (Figure 1), similar to the results of Ramanathan et al. (2011). Variability in BC prediction by PR method as shown by $95 \%$ confidence interval (dashed lines in Figure 1) was less in combined cities calibration than individual city.

Change in RMSE/(Mean EC) of a particular city data set, when calibration other than its own was used to predict $\mathrm{BC}$ by PR method, remained within $\pm 10 \%$ of the value observed when city's own calibrations was applied. Site-specific calibrations for LA, Denver and Riverside when applied to total US test samples $(\mathrm{LA}+$ Denver + Riverside $=216)$ resulted in average absolute error $\left(\mu \mathrm{g} \mathrm{cm}^{-2}\right)$ of $1.33,1.33$ and 1.20, respectively, and average of RMSE/ Mean EC for the same were $0.29,0.30$ and 0.27 , respectively which shows that LA and Denver calibration are almost equivalent, and Riverside calibration had slightly less error ( 3\% RMSE relative to mean EC) (Table 1). This may be due to similar particulate matter (PM) sources at the three locations, which are mainly attributed to vehicular and industrial emissions (Liu et al., 2005). Therefore, the difference in $\mathrm{BC}$ estimates by PR method using calibrations prepared at different locations was not significant enough to recommend the use of site-specific calibrations, provided the reference EC measurement method used for creating calibrations remains same.

A sensitivity test was performed to examine the effect of increasing the number of sample points used in the calibration on the error in $\mathrm{BC}$ estimate by $\mathrm{PR}$ method. Calibrations prepared with varying number of filter samples $(n=40,70,100$ and 150$)$ were applied on a test data set $\left(\mathrm{n}=183\right.$; mean $\left.\mathrm{EC} \pm \mathrm{SD}=6.39 \pm 4.67 \mu \mathrm{g} \mathrm{cm}^{-2}\right)$ and compared with original $\mathrm{EC}$ measurements. A marginal decrease of 3\% (RMSE relative to mean EC) was observed when the number of samples was increased from 40 to 100 whereas no change was observed when number of samples were increased further to 150 (Table S1; Supporting Material, page S3). This suggests 
that increasing the number of data points in the calibration has little effect in decreasing the error and close to 40 sample points would be suitable for creating the calibration.

\subsection{Season specific calibrations}

Strong correlations between EC loading and red reflectance were found for spring $\left(\mathrm{R}^{2}=\right.$ $0.85)$, fall $\left(\mathrm{R}^{2}=0.81\right)$ and winter $\left(\mathrm{R}^{2}=0.87\right)$ but relatively weaker for summer $\left(\mathrm{R}^{2}=0.67\right)$ (Figure 2). The EC loading range covered in the calibrations was highest for winter (1.10-30.07) $\mu \mathrm{g} \mathrm{cm}^{-2}$

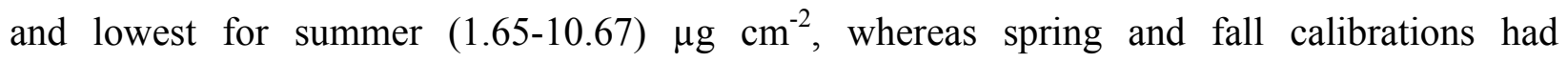
comparable loading ranges, which are (0.38-16.31) $\mu \mathrm{g} \mathrm{cm}^{-2}$ and (1.54-18.69) $\mu \mathrm{g} \mathrm{cm}^{-2}$, respectively. Calibrations were compared to each other by applying them across different season's test data sets in the same manner as described in section 2.4. For all seasons, the RMSE/Mean EC observed was least or nearly equal to smallest (within 1\%) when $\mathrm{BC}$ is predicted using the calibration prepared in the same season ("best case scenario") by PR method.

For summer test samples $\left(\mathrm{n}=48\right.$, mean $\left.\mathrm{EC} \pm \mathrm{SD}=5.00 \pm 2.67 \mu \mathrm{g} \mathrm{cm}^{-2}\right)$, each season's calibration performance was nearly the same, with an RMSE/(Mean EC) of $0.20 \pm 0.01$. On the other hand, in the case of fall $\left(\mathrm{n}=43\right.$, mean $\left.\mathrm{EC} \pm \mathrm{SD}=9.18 \pm 5.29 \mu \mathrm{g} \mathrm{cm}^{-2}\right)$ and winter test samples ( $\mathrm{n}=39$, mean $\mathrm{EC} \pm \mathrm{SD}=10.28 \pm 8.10 \mu \mathrm{g} \mathrm{cm}{ }^{-2}$ ), using a summer calibration resulted in an error of $60 \%$ and $40 \%$ (percent RMSE relative to mean EC), respectively, as opposed to $30 \%$ and $20 \%$ when season specific calibrations were applied (Table 2). This may be due to the weaker correlation observed for summer samples $\left(\mathrm{R}^{2}=0.67\right)$ or due to difference between the EC loading range observed in summer calibration compared to that of fall and winter test samples. Winter calibration having highest EC loading range resulted in $32 \%$ and $36 \%$ error (percent 
RMSE relative to mean EC) when applied to the spring and fall test samples ( $n=47$, mean $\mathrm{EC} \pm$ $\mathrm{SD}=4.83 \pm 3.59 \mu \mathrm{g} \mathrm{cm}^{-2}$ ), whereas the same for summer and winter test samples was $20 \%$ (Table 2). This disagreement between calibrations of the PR method prepared in different seasons could be due to changes in optical properties of Black Carbon aerosol sampled, as PR method is based on optical reflectance of photographs of aerosol loaded filters, or due to differences in the range of EC loading sampled in different seasons. Specifically, Fall and Winter filters were more heavily loaded, with maximum loading values of 18.7 and $30.1 \mu \mathrm{g} \mathrm{cm}^{-2}$, respectively, compared to Spring and Summer maximum values of 16.3 and $10.7 \mu \mathrm{g} \mathrm{cm}^{-2}$, respectively. This indicates that using a calibration prepared from samples of different season could result in high error when estimating BC by PR method and a season-specific calibration can be used to minimize the error.

\subsection{Site-season specific calibrations}

Each season calibration was further separated into different cities: LA, Denver and Riverside to create site-season specific calibrations to investigate differences in the calibration for each season as a function of sites. Three cities calibrations for each season were cross applied on the data sets used for creating calibrations to examine the difference between the three cities calibration in each season (Bland and Altman, 1986). For spring and summer, insignificant differences occurred in the RMSE/Mean EC and average absolute error between the three cities calibrations. For Fall_LA data set, RMSE/Mean EC was $\sim 2$ times more when Fall_LA calibration was used rather than Fall_Denver and vice versa for Fall_Denver data set. Also, for Winter_LA data set, RMSE/Mean EC was more than 2 times when Winter_Denver calibration was used rather than Winter_LA to predict BC by PR method (Table S2; Supporting Material). 
This shows that for Fall and Winter samples, LA and Denver calibrations resulted in high errors when applied across each other data sets. This is consistent with the results of season-specific calibrations where high errors were observed in fall and winter season calibrations, due to higher EC surface loadings as compared to spring and summer seasons.

\subsection{Method specific calibration}

The PR calibration prepared from all US samples ("US combined") was used to predict $\mathrm{BC}\left(\mu \mathrm{g} \mathrm{cm}^{-2}\right)$ for Kanpur test samples $(\mathrm{n}=32)$. RMSE/(Mean EC) between the BC estimates using "US combined calibration" of the PR method and thermo-optical EC measurements was found to be 0.50 whereas average absolute error for the same was $0.94 \mu \mathrm{g} \mathrm{cm}^{-2}$, respectively (Table 3). The reason for this large error in prediction could be the difference in the protocol followed in EC measurements of the calibration used (IMPROVE) and test data (NIOSH) as large disagreements have been found between the two protocols (Chow et al., 2001). Also, PM sources at the two locations are slightly different with US samples exposed to vehicular and industrial emissions (Liu et al., 2005) whereas Kanpur samples also have biomass burning which is found to be significant in winter season (Kaul et al., 2011).

Calibration prepared using India urban filter samples $(n=40$; mean $\mathrm{EC} \pm \mathrm{SD}=2.09 \pm 1.51$ $\mu \mathrm{g} \mathrm{cm}^{-2}$ ) where EC was analyzed following TO NIOSH protocol had EC loading in the range $(0.48-8.47) \mu \mathrm{g} \mathrm{cm}^{-2}$ (Figure 3), was used to predict BC for Kanpur test data samples. RMSE/(Mean EC) was reduced from 0.50 in case of "US combined" calibration to 0.24 , whereas average absolute error was reduced from $0.94 \mu \mathrm{g} \mathrm{cm}^{-2}$ to $0.36 \mu \mathrm{g} \mathrm{cm}^{-2}$, when method-specific calibration (India urban) was applied (Table 3). India urban calibration when applied to US samples $\left(\mathrm{n}=333\right.$, Mean $\left.\mathrm{EC} \pm \mathrm{SD}=7.09 \pm 5.34 \mu \mathrm{g} \mathrm{cm}^{-2}\right)$ resulted in very high error of $63 \%$ (RMSE relative to Mean EC) due to large difference in the range of EC surface loadings between 
the samples collected in India $\left(n=72\right.$, Mean $\left.E C \pm S D=2.05 \pm 1.51 \mu \mathrm{g} \mathrm{cm}^{-2}\right)$ and US $(n=333$, Mean $\mathrm{EC} \pm \mathrm{SD}=7.09 \pm 5.34 \mu \mathrm{g} \mathrm{cm}^{-2}$ ). This shows that calibration of the PR method depends largely on the EC measurement method used for creating the calibration, and also on the range of EC surface loadings sampled. Therefore, the use of method specific calibration could reduce the error in BC prediction significantly.

\subsection{Piece-wise regression analysis}

An example of a three-part quadratic regression based on linear determination of the "knots" is presented in Figure 4, to illustrate the results of one variation of the piece-wise analysis. For the combined US urban data set, piece-wise regressions improved the estimation of $\mathrm{BC}$ only slightly, reducing the RMSE/(Mean EC) from 0.27 to 0.26 for a three-part quadratic regression based on the linear determination of the location of the knots. The knots were located at red pixel values of 86 and 136, corresponding to loading values of 17.6 and $5.8 \mu \mathrm{g} \mathrm{cm}^{-2}$. Linear and exponential fits for these and different knot locations did not improve the results. For the India data, piece-wise regressions improved the estimation of BC from an RMSE/(Mean EC) of 0.32 to $0.28 \mu \mathrm{g} \mathrm{cm}^{-2}$ for a two-part quadratic regression based on the linear determination of the location of the knot. The knot occurred at a red pixel value of 169 , corresponding to a loading of $2.2 \mu \mathrm{g} \mathrm{cm}^{-2}$. Data from the original Ramanathan et al. (2011) paper were also analyzed (data not shown) and for every variation that included three regressions for both the US and original data, high loading values, corresponding to red pixel values $<90$, were isolated by a knot for separate regression. The India data did not contain such high loading values, with the lowest red pixel value of 99 . These results illustrate the effective loading range of the 
photographic method, where high loading values above $20 \mu \mathrm{g} \mathrm{cm}^{-2}$, with relatively dark filters have a greater error.

\section{Conclusion}

Difference in BC estimates among calibrations from LA, Denver and Riverside was not significant enough to recommend the use of site-specific calibration of the PR method, and a combined urban US calibration can be used to supplement large scale monitoring of TO-EC concentration at other urban locations. Optimum number of sample points to create a calibration was found to be $\sim 40$, as increasing the points further had little effect on the estimated BC error. Season based calibrations resulted in least error when applied to the same season. However, due to difference in the loading range of EC sampled in different seasons, high errors up to $60 \%$ RMSE relative to mean EC, was observed in the case of summer calibration. Thus, the use of season-specific calibration is recommended for minimizing the error in estimating BC by PR method. Although, urban US combined calibration resulted in percent RMSE/Mean EC of 50\% when applied to urban India test samples, but a method and source specific calibration of India urban reduced the error to $24 \%$. Therefore, calibration of the PR method depends largely on reference $\mathrm{BC}$ measurement methods and a method-specific calibration can be used to reduce the error significantly. Piece-wise regressions only slightly improved results for either data set, possibly not warranting the routine use of this complicated analysis. However, the three-part, piece-wise regressions also consistently isolated the high loading values to improve the fits. This suggests an upper loading limit to this method and supports the observation that very dark filters with loading $>20 \mu \mathrm{g} \mathrm{cm}^{-2}$ are effectively "black" and are not as suitable to the photographic method analysis as are lighter loadings. At the other extreme, filter loadings $<\sim 2 \mu \mathrm{g} \mathrm{cm}^{-2}$ do not 
have sufficient loadings for the photographic method to have a signal that is reliably differentiate from variability in the measurements of blank filters. In addition, samples with less than a $0.1 \mu \mathrm{g}$ $\mathrm{cm}^{-2}$ for a single red pixel value so should also be avoided.

\section{Acknowledgement}

The authors wish to acknowledge the Ministry of Human Resource and Development (MHRD) for funding the effort of IIT-Kanpur on this research project. We are also thankful to Dr. Philip Fine at South Coast Air Quality Management District (SCAQMD) and Dr. Bradley Rink at the Colorado Department of Public Health and Environment, Air Pollution Control Division for providing filter samples for the project. Support for this work was provided by the U.S. Department of Energy (Grant \# DE-SC0008205).

\section{Supporting Material Available}

Average absolute error and RMSE/Mean EC between EC $\left(\mu \mathrm{g} \mathrm{cm}^{-2}\right)$, measured using thermooptical (TO) EC-OC analyzer and BC measured using PR method calibrations with varying number of samples used: $n=40,70,100$ and 150 (Table S1), Average absolute error and RMSE/Mean EC between EC $\left(\mu \mathrm{g} \mathrm{cm}^{-2}\right)$ measured using thermo-optical EC-OC analyzer and BC measured using site-season-specific calibrations of PR method (Table S2). This information is available free of charge via the Internet at http://www.atmospolres.com. 


\section{References}

Bland, J. M., Altman, D. G., 1986. Statistical methods for assessing agreement between two methods of clinical measurement. Lancet 1, 307-310.

Bodhaine, B. A.,1995. Aerosol absorption measurements at Barrow,Mauna Loa and the South Pole. Journal of Geophysical Research 100, 8967-8975.

Chow, J. C., Watson, J. G., Crow, D., Lowenthal, D. H., Merrifield, T.,2001. Comparison of IMPROVE and NIOSH Carbon Measurements. Aerosol Science and Technology 34, 23-34.

Cross, E. S., Onasch, T. B., Ahern, A., Wrobel, W., Slowik, J., G., Olfert, J., Lack, D. A., Massoli, P., Cappa, C. D., Schwarz, J. P., Spackman, J. R., Fahey, D. W., Sedlacek, A., Trimborn, A., Jayne, J. T., Freedman, A., Williams, L. R., Ng, N. L., Mazzoleni, C., Dubey, M., Brem, B., Kok, G., Subramanian, R., Freitag, S., Clarke, A., Thornhill, D., Marr, L. C., Kolb, C. E., Worsnop, D. R., Davidovits, P.,2010. Soot Particle Studies-Instrument Inter-Comparison-Project Overview. Aerosol Science and Technology 44, 592-611.

Ezzati, M., Kamen, D.M., 2002. The health impact of exposure to indoor air pollution from solid fuels in developing countries: knowledge, gaps and data needs. Environmental Health Perspectives 110 , 1057-1068.

Grieshop, A. P., Reynolds, C. C. O., Kandlikar, M.,Dowlatabadi, H., 2009. A Black-Carbon Mitigation Wedge. Nature Geoscience 2, 533-534.

Gupta, T., Jaiprakash, Dubey, S., 2011. Field Performance Evaluation of a newly developed PM2.5 Sampler at IIT Kanpur. Science of the Total Environment 409, 3500-3507.

Hansen, A. D. A., Rosen, H., Novakov, T., 1984. The aethalometer: An instrument for the real-time measurement of optical absorption by aerosol particles. Science of the Total Environment 36, 191-196.

Hitzenberger, R., Petzold, A., Bauer, H., Ctyroky, P., Pouresmaeil, P., Laskus, L. and Puxbaum, H., 2006.Intercomparison of Thermal and Optical Measurement Methods for Elemental Carbon and Black Carbon at an Urban Location. Environmental Science and Technology 40, 6377-6383.

Jacobson, M. Z., 2001. Strong Radiative Heating due to the Mixing State of Black Carbon in Atmospheric Aerosols. Nature 409, 695-697.

Kaul, D. S., Gupta, T., Tripathi, S. N., Tare, V.,Collett Jr, J. L., 2011. Secondary organic aerosol: A comparison between foggy and non-foggy days. Environmental Science and Technology 45, 7307-7313.

Liu, W., Wang, Y., Russell, A., Edgerton, E. S., 2005.Atmospheric aerosol over two urban-rural pairs in the southeastern United States: Chemical composition and possible sources. Atmospheric Environment 39, 4453-4470.

NIOSH, 1996. Elemental carbon (diesel particulate) method 5040. NIOSH Manual of Analytical Methods, fourth ed. National Institute for Occupational Safety and Health, Cincinnati, Ohio (1st Suppl.).

Pope III, C., Burnett, R. T., Thun, M. J.,Calle, E. E., Krewski, D., Ito, K., Thurston, G.D., 2002. Lung cancer, cardiopulmonary mortality, and long-term exposure to fine particulate air pollution. Journal of the American Medical Association, 287, 1132-1141.

Ramanathan, V., Carmichael, G., 2008.Global and Regional Climate Changes due to Black Carbon. Nature Geoscience. 1, 221-227.

Ramanathan, V., Feng, Y., 2009. Air Pollution, Greenhouse Gases and Climate Change: Global and Regional Perspectives. Atmospheric Environment 43, 37-50. 
Ramanathan, N., Lukac, M., Ahmed, T., Kar, A., Praveen, P. S., Honles, T., Leong, I., Rehman, I. H., Schauer, J. J., Ramanathan, V., 2011. A cellphone based system for large scale monitoring of black carbon. Atmospheric Environment 45, 4481-4487.

Samet, J. M., Dominici, F., Curriero, F. C., Coursac, I., Zeger, S. L., 2000. Fine Particulate Air Pollution and Mortality in 20 U.S. Cities, 1987-1994. New England Journal of Medicine 343, 1742-1749.

Schmid, H., Laskus, L., Abraham, J. H., Baltensperger, U., Lavanchy, V., Bizjak, M., Burba, P., Cachier, H., Crow, D., Chow, J., Gnauk, T., Even, A., Brink, H. M. T, Giesen, K. P., Hitzenberger, R., Hueglin, C., Maenhaut, W., Pio, C., Carvalho, A., Putaud, J. P., Toom, S. D., Puxbaum, H., 2001. Results of the "carbon conference" international aerosol carbon round robin test stage I.

Atmospheric Environment 35, 2111-2121.

Schwartz, S. E., Buseck, P. R.,2000. Absorbing Phenomena. Science 288, 989-990. 


\section{Figure captions}

Figure 1. Correlation of red reflectance with EC loading on filters as measured using thermooptical (TO) EC-OC analyzer following IMPROVE protocol for samples collected in A) LA, B) Denver, C) Riverside and D) US combined (LA, Denver and Riverside). Solid line is best fit and two dashed lines are $95 \%$ confidence bands.

Figure 2. Correlation of red reflectance with EC loading on filters as measured using thermooptical (TO) EC-OC analyzer for different seasons: A) Summer, B) Spring, C) Fall and D) Winter. Solid line is best fit and two dashed lines are 95\% confidence bands.

Figure 3.Correlation of red reflectance with EC loading on filters as measured using the thermooptical (TO) EC-OC analyzer following NIOSH protocol for A) India urban samples, and IMPROVE protocol for B) US urban samples.

Figure 4. Three-part quadratic piece-wise fit of the US combined data with "knot" locations indicated by vertical lines. 


\section{Figures}

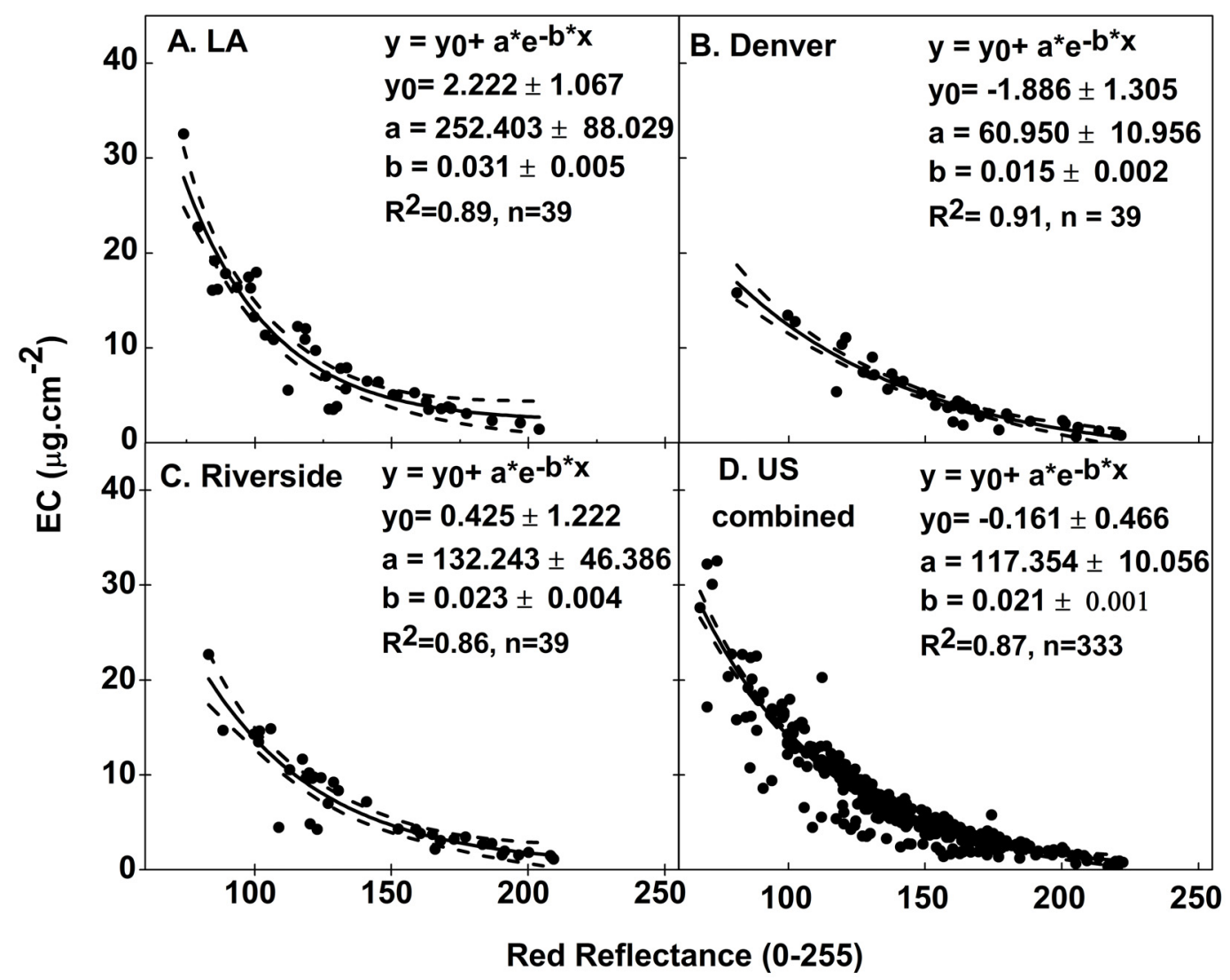

Figure 1. 


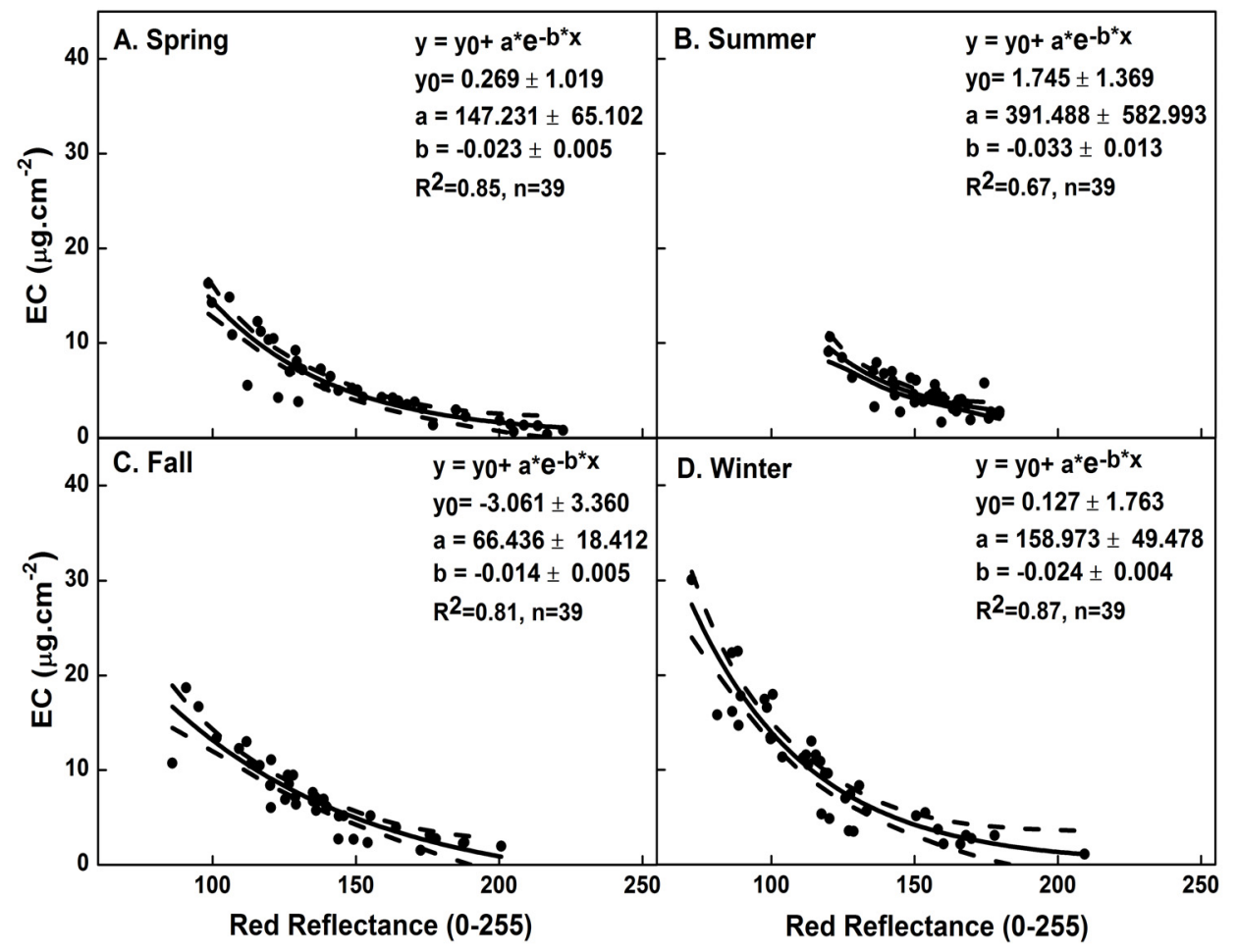

Figure 2. 


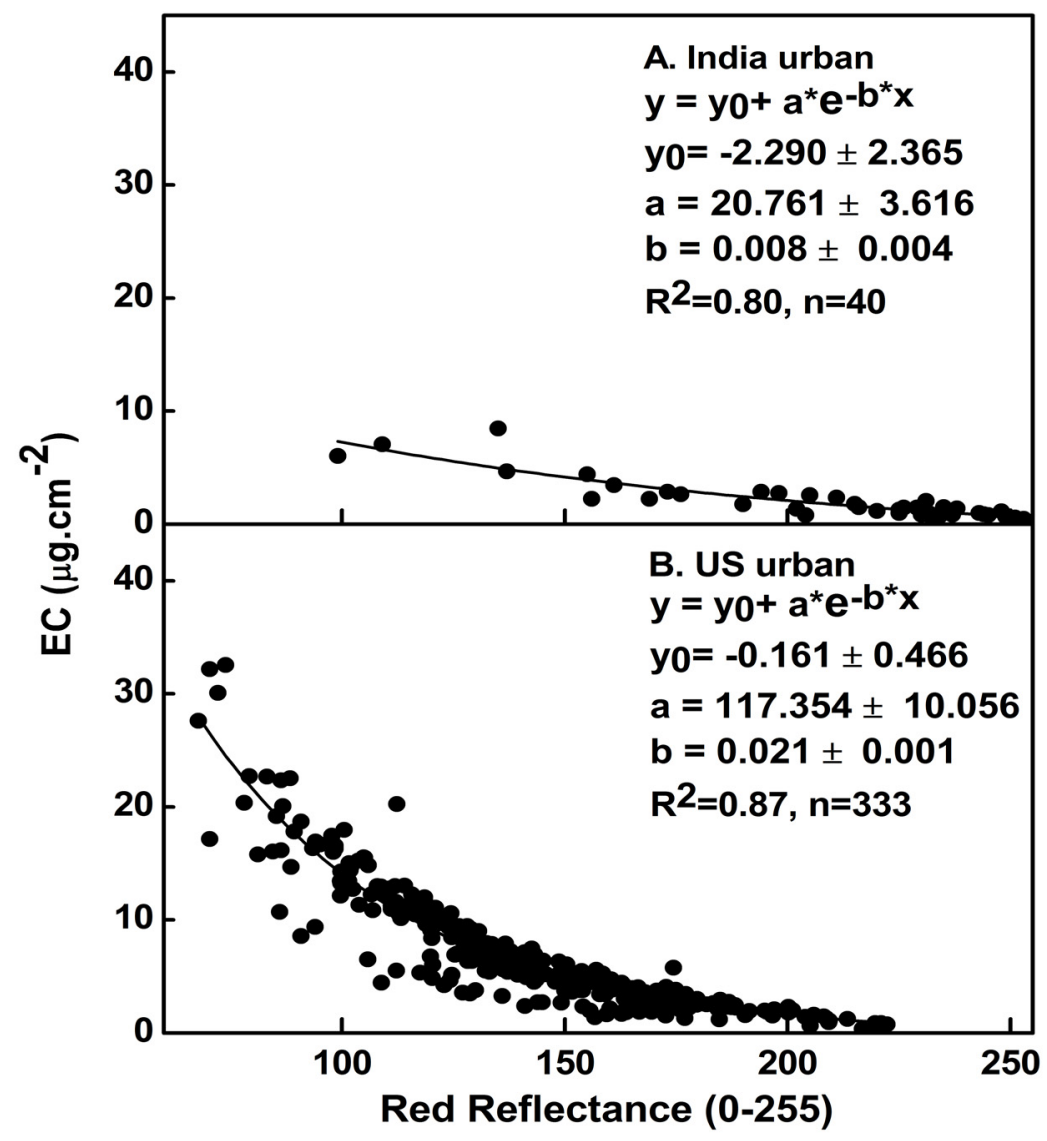

Figure 3. 


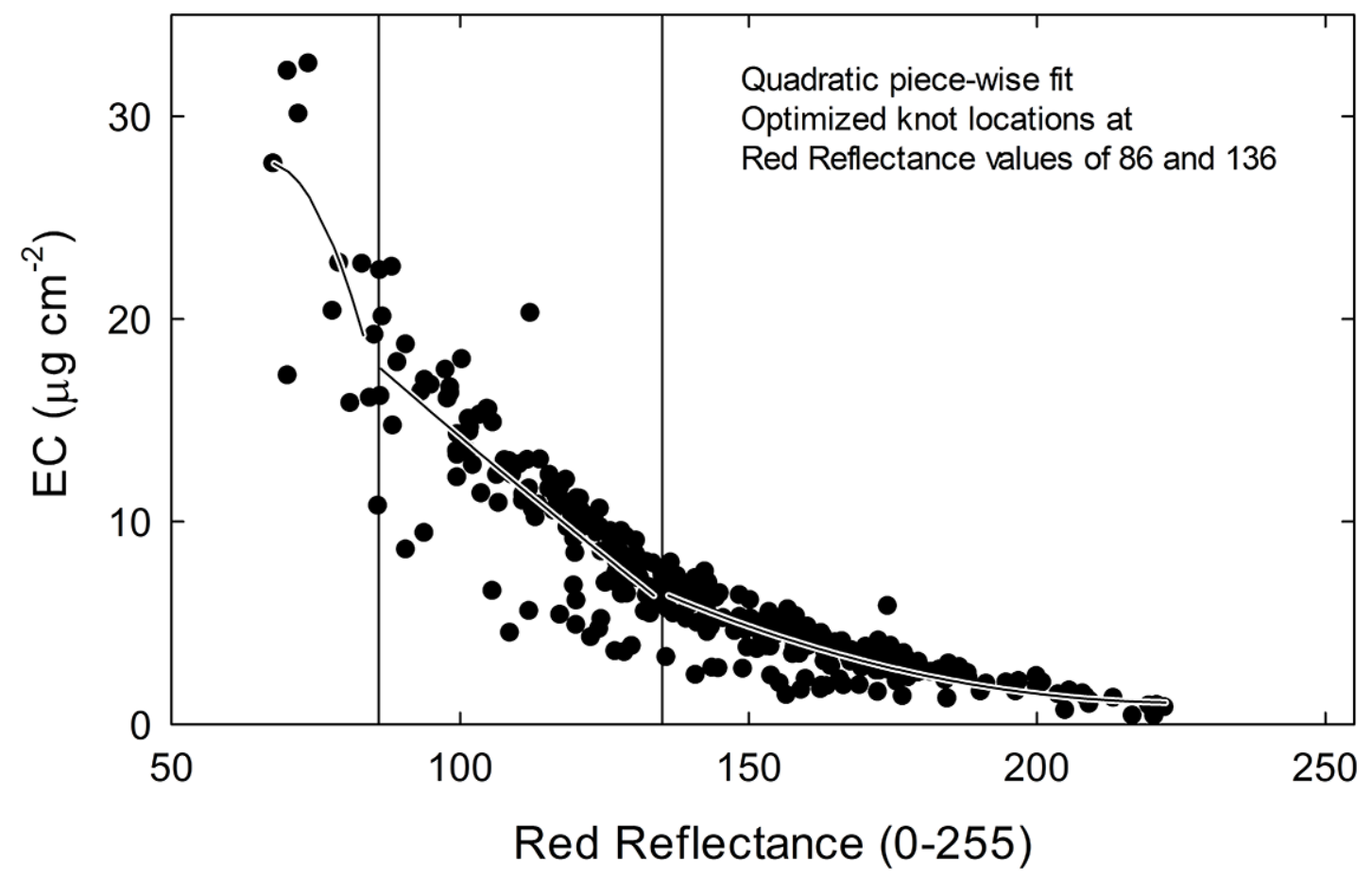

Figure 4 
$\underline{\text { Tables }}$

Table 1: Average absolute error and RMSE/Mean EC between EC measured using thermo-optical (TO) EC-OC analyzer and BC measured using PR method calibrated to location specific calibrations for LA ( $n=39$; mean \pm SD of $\left.9.58 \pm 6.91 \mu \mathrm{g} \mathrm{cm}^{-2}\right)$, Denver $(n=39$; mean \pm SD of $5.02 \pm 3.67$ $\left.\mu \mathrm{g} \mathrm{cm}^{-2}\right)$ and Riverside $\left(\mathrm{n}=39\right.$; mean \pm SD of $\left.6.87 \pm 5.10 \mu \mathrm{g} \mathrm{cm}^{-2}\right)$.

\section{Calibrations}

Average Absolute Error $\quad$ RMSE/Mean EC $\left(\mu \mathrm{g} \mathrm{cm}^{-2}\right)$

Test Data

(Mean EC \pm SD,

n)

$\left(\mu \mathrm{g} \mathrm{cm}^{-2}\right)$

LA

$(8.33 \pm 5.87,77)$

Denver

1.05

1.02

0.97

0.24

0.25

0.23

$(5.83 \pm 3.63,57)$

Riverside

$(6.72 \pm 5.15,82)$

$$
1.49
$$

1.37

1.22

0.33

0.33

0.30

$\begin{array}{ccccccc}\text { Average } \pm \text { SD } & 1.33 \pm & 1.33 \pm & 1.2 \pm & 0.29 \pm & 0.30 \pm & 0.27 \pm \\ & 0.24 & 0.30 & 0.22 & 0.05 & 0.04 & 0.04\end{array}$


Table 2: Average absolute error and RMSE/Mean EC between EC $\left(\mu \mathrm{g} \mathrm{cm}^{-2}\right)$ measured using thermo-optical (TO) EC-OC analyzer and BC measured using PR method calibrated to season-specific calibrations for samples collected in Spring $\left(n=39\right.$; mean EC \pm SD of $\left.5.86 \pm 4.17 \mu \mathrm{g} \mathrm{cm}^{-2}\right)$, summer $(n=39$; mean EC \pm SD of 4.73 $\left.\pm 2.10 \mu \mathrm{g} \mathrm{cm}^{-2}\right)$, fall $\left(n=39\right.$; mean EC \pm SD of $\left.7.21 \pm 4.04 \mu \mathrm{g} \mathrm{cm}^{-2}\right)$ and winter $(n=39$; mean EC \pm SD of $10.40 \pm 6.65 \mu \mathrm{g} \mathrm{cm}^{-2}$ ).

\section{Calibrations}

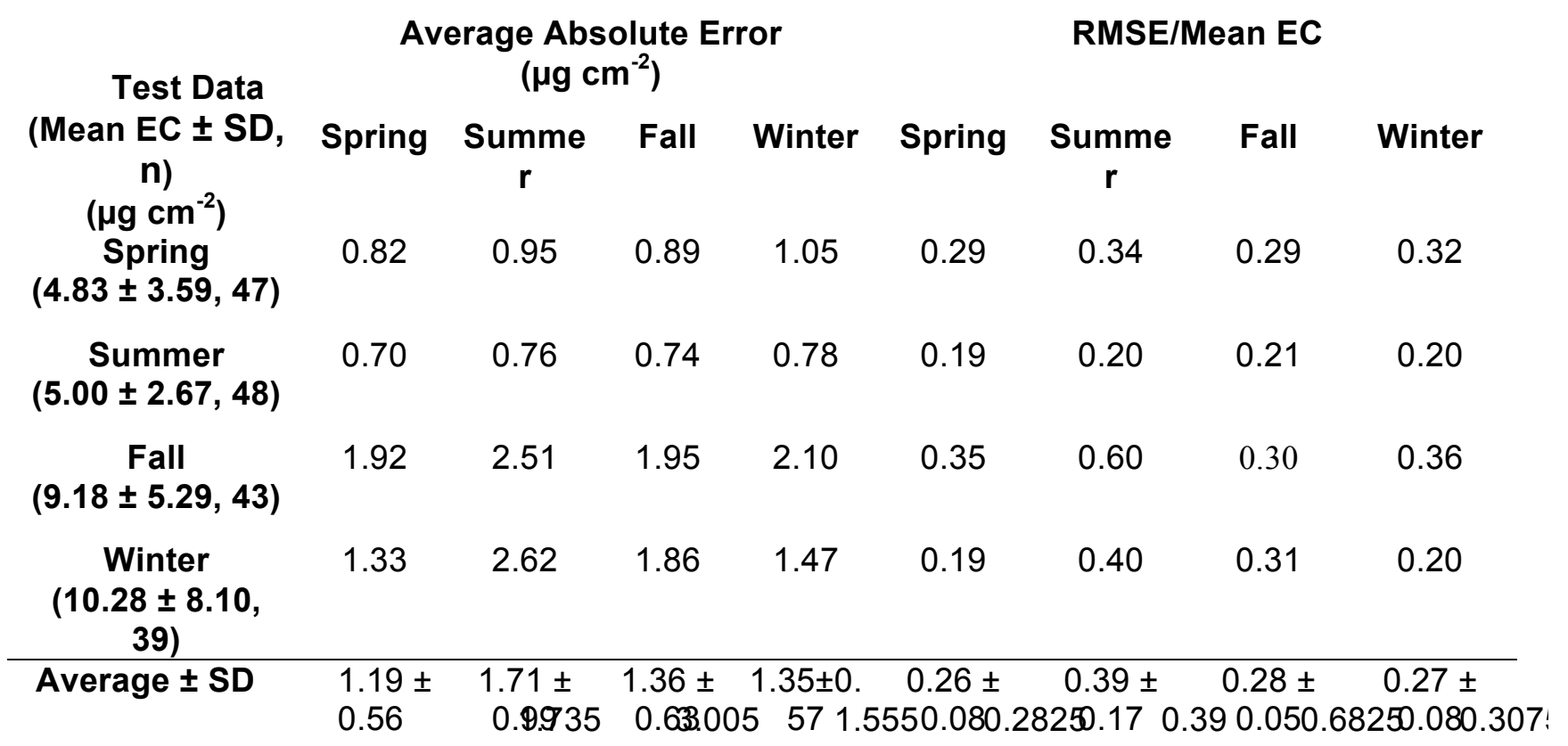


Table 3: Average absolute error and RMSE/Mean EC between EC ( $\mu \mathrm{g}$ $\mathrm{cm}^{-2}$ ) measured using thermo-optical (TO)EC-OC analyzer and BC measured using PR method calibrations from samples collected in Kanpur ( $\mathrm{n}=40$; mean $\mathrm{EC} \pm \mathrm{SD}=2.01 \pm 1.81 \mu \mathrm{g} \mathrm{cm}$ ) and combined urban locations in US (LA, Denver and Riverside $(n=333$; mean EC \pm SD of $7.09 \pm 5.34 \mu \mathrm{g} \mathrm{cm}^{-2}$ ).

\section{Calibrations}

\begin{tabular}{|c|c|c|c|c|}
\hline \multirow{2}{*}{$\begin{array}{l}\text { Test Data } \\
\text { (Mean EC } \pm \text { SD, } \\
\text { n) } \quad \\
\quad\left(\mu \mathrm{g} \mathrm{cm}^{-2}\right)\end{array}$} & \multicolumn{2}{|c|}{$\begin{array}{l}\text { Average Absolute } \\
\text { Error }\left(\mu \mathrm{g} \mathrm{cm}^{-2}\right)\end{array}$} & \multicolumn{2}{|c|}{ RMSE/Mean EC } \\
\hline & $\begin{array}{c}\text { US } \\
\text { combined }\end{array}$ & Kanpur & $\begin{array}{l}\text { US } \\
\text { combined }\end{array}$ & Kanpur \\
\hline $\begin{array}{c}\text { Kanpur } \\
(2.01 \pm 1.05,32)\end{array}$ & 0.94 & 0.36 & 0.50 & 0.24 \\
\hline
\end{tabular}

